Les actes de colloques du musée du quai Branly Jacques Chirac

$7 \mid 2016$

Nathan Wachtel. Histoire et anthropologie

\title{
La condition marrane : un fait social total à l'épreuve de la longue durée
}

Natalia Muchnik

\section{(2) OpenEdition \\ Journals}

Édition électronique

URL : http://journals.openedition.org/actesbranly/724

ISSN : 2105-2735

Éditeur

Musée du quai Branly Jacques Chirac

Référence électronique

Natalia Muchnik, «La condition marrane : un fait social total à l'épreuve de la lonque durée », Les actes de colloques du musée du quai Branly Jacques Chirac [En ligne], 7 | 2016, mis en ligne le 15 novembre 2016, consulté le 08 septembre 2020. URL : http://journals.openedition.org/actesbranly/724

Ce document a été généré automatiquement le 8 septembre 2020

(c) Tous droits réservés 


\title{
La condition marrane : un fait social total à l'épreuve de la longue durée
}

\author{
Natalia Muchnik
}

\section{Mme Natalia MUCHNIK}

Merci Carmen. Je tiens tout d'abord à remercier les organisateurs de m'avoir invitée à cette rencontre autour de l'œuvre de Nathan WACHTEL. Il n'est pas anodin pour moi d'y participer en évoquant l'un de nos intérêts communs, en l'occurrence les marranes. Comme vous le savez, il s'agit d'un terme controversé, mais établi dans l'historiographie désignant les chrétiens d'origine juive (les judéoconvers) accusés de judaïser en secret par les inquisitions ibériques - ainsi que par les tribunaux italiens que je n'évoquerai pas ici.

2 Je tiens à souligner que le terme «marrane " n'est pas utilisé par les acteurs, dans la péninsule Ibérique (davantage dans le reste de l'Europe), mais qu'il a été imposé par l'historiographie. Lorsque Nathan WACHTEL a publié le premier volume de sa trilogie marrane La foi du souvenir, en 2001,j'achevais pour ma part ma thèse de doctorat sur un marrane du XVII ${ }^{\mathrm{e}}$ siècle, Juan de Prado, converti au judaïsme à Amsterdam. Depuis, deux autres volumes sont parus: l'un, centré sur les rapports des marranes aux tribunaux inquisitoriaux, à savoir La logique des bûchers ; et ce, tout particulièrement au Brésil au début du XVIII ${ }^{\mathrm{e}}$ siècle. L'autre ouvrage, publié en 2011 et intitulé Mémoires marranes, portait sur les résurgences du marranisme dans le Brésil contemporain et constituait le résultat non tant d'une enquête ethnographique que d'une enquête d'histoire orale, comme Nathan WACHTEL l'indique lui-même en introduction. Pour ma part, j'ai poursuivi mes travaux sur la construction de l'identité marrane dans l'Espagne dite moderne, c'est-à-dire aux XVI ${ }^{\mathrm{e}}, \mathrm{XVII}^{\mathrm{e}}$ et XVIII ${ }^{\mathrm{e}}$ siècles. Toute la difficulté est donc ici, pour moi, de synthétiser les apports de l'approche de Nathan WACHTEL pour l'historienne que je suis et pour les études marranes plus généralement, mais également les questions qu'elles soulèvent.

3 Je commencerai par une citation: «Entre mémoire et oubli, la condition marrane s'accompagne au fil du temps de représentations et réactions ambivalentes, tant positives que négatives, à l'égard de l'héritage juif: soit la foi du souvenir et la 
vénération des martyrs, soit le déni des ancêtres qui ont transmis à leurs descendants le stigmate de leur sang impur ».

4 C'est par ces termes que Nathan WACHTEL débute Mémoires marranes, itinéraires dans le sertão $d u$ Nordeste brésilien, troisième volet de sa trilogie. Nous retrouvons dans ce passage liminaire les deux aspects parcourant l'ensemble des volumes, c'est-à-dire, d'une part, la mémoire et l'oubli, son corollaire, envisagé comme une pratique mémorielle à part entière et, d'autre part, le rapport à la judéité ou, plus largement, les rapports entre mémoire et identité. Ce sont ces deux aspects qui retiendront mon attention aujourd'hui. Je m'attacherai ainsi, pour commencer, à la place de la mémoire ou du "devoir de mémoire», selon les termes de Nathan WACHTEL, dans la construction identitaire marrane et leur constitution en tant que groupe. Puis, j'évoquerai le problème des sources inquisitoriales, matériau central dans les travaux de Nathan WACHTEL et les études marranes en général. Je terminerai, enfin, sur la question du marranisme comme « fait social total » et de son inscription dans la longue durée.

5 I. La mise en avant de la mémoire des origines est essentielle pour comprendre la construction de l'identité marrane. Nous assistons en effet à deux mouvements concomitants et indissociables. L'un, du côté des sociétés ibériques, l'autre dans la société marrane. Du côté de la société d'accueil hispanique et portugaise, comme vous le savez, les statuts de pureté de sang (limpieza de sangre, en espagnol) sont adoptés par les institutions ibériques à partir du XVI ${ }^{\mathrm{e}}$ siècle et instaurent une frontière interne au sein de la population opposant anciens et nouveaux chrétiens. Les nouveaux chrétiens sont les convertis aussi bien d'origine juive que d'origine musulmane et leurs descendants.

6 Il est important de noter que cette scission dépasse le cadre du religieux, puisqu'elle s'appuie sur le sang considéré comme impur (manchado, en espagnol) des nouveaux chrétiens marqués par l'infamie et suspects d'être des hérétiques en puissance. Cette scission est restée en vigueur jusqu'au XVIIIe ${ }^{\mathrm{e}}$ voire jusqu'au XIX ${ }^{\mathrm{e}}$ siècle. Cela est dû à plusieurs dispositifs: les sambenitos (morceaux de toiles suspendus dans les églises portant le nom des condamnés); les dits « livres verts » recensant les lignages impurs et les linajudos, soit les férus de généalogie enquêtant et monnayant souvent leur silence. Ainsi, la stigmatisation des judéoconvers est ainsi double, à la fois liée au sang et à la condamnation par le Saint-Office. Il faut noter que le critère de la pureté est devenu un classificateur aussi important que la noblesse (l'hidalguía, en espagnol) en particulier en Espagne avec l'entrée de nombreux judéoconvers dans la petite noblesse.

7 Il convient de souligner à ce titre la spécificité des statuts de pureté de sang: d'une part, au Portugal où les nouveaux chrétiens, les judéoconvers, constituent un groupe de pression, une forme de lobby ayant des représentants et pouvant négocier avec la monarchie et rassembler de fortes sommes, pour les pardons généraux en particulier ; d'autre part, dans les colonies américaines du fait des métissages et du système des castas. D'ailleurs, cet aspect n'est pas très présent dans la trilogie. Il s'agit d'ailleurs là d'une question qui pourrait être discutée, notamment dans le cas du Brésil où les judéoconvers constituent une partie importante de la population dite "blanche». Par conséquent, le stigmate de ladite "pureté de sang » est d'une certaine manière atténué d'autant qu'ils sont souvent membres des élites et que les mariages entre judéoconvers et vieux-chrétiens sont fréquents. À mon avis, cette singularité a sans doute des 
conséquences en termes d'identification pour les marranes et pour la cohésion du groupe.

8 En effet, la stigmatisation du sang et la séparation d'une partie de la population, les nouveaux-chrétiens, participe de la constitution du groupe stigmatisé, en l'occurrence les judéoconvers, que les sociétés ibériques désignent par des termes collectifs et englobants. L'incidence en est accentuée chez ceux que l'on qualifie de cryptojudaïsants ou marranes.

9 Du côté des marranes justement, cette mémoire des origines et des traditions, à la fois individuelles et collectives, joue un rôle à plusieurs niveaux, suivant deux types de mémoire. La première, "mémoire d'origine ", légitime le groupe et lui assure une continuité d'existence, suscitant chez les marranes l'élaboration d'un corpus mythologique bigarré suivant un processus qui a tout du "renversement du stigmate » étudié par Erving GOFFMAN. En effet, loin de sortir de la classification établie par les sociétés ibériques, qui les stigmatise en désignant leur sang comme impur, les marranes s'approprient et inversent la catégorisation cristallisée en créant leur propre sociodicée. Ainsi, si les marranes se perçoivent et sont perçus comme étrangers -à la fois comme nouveaux-chrétiens et, pour beaucoup en domaine hispanique, d'origine portugaise ou du moins désignés comme tels dans le monde hispanique-, ils se déterminent comme des autochtones. .D'ailleurs, nous parlions de l'autochtonie qui est une constante. Ils s'affirment comme les vrais nobles au sang transfiguré par l'antiquité du peuple juif. Cela les amène à s'assimiler aux Basques qui sont les nobles et les vieux chrétiens par excellence en domaine hispanique. Par conséquent, ils constituent une sorte de noblesse transfigurée par l'antiquité du peuple juif. Cette noblesse ancestrale s'associant à l'affirmation d'une pureté de sang spécifiquement juive développe un " orgueil de lignage» que nous retrouvons dans l'ensemble de la diaspora, alors appelée la Nação. Cette diaspora judéo-ibérique se distingue d'autres groupes juifs, notamment les germaniques (plus tard ashkénazes). Cette distinction est très importante. Cet « orgueil de lignage » que nous pourrions qualifier « d'hidalguía marrane " participe d'ailleurs d'une forme d'éthnicisation de la judéité (et du marranisme), qui va bien au-delà du religieux au sens contemporain du terme.

Ensuite, une seconde forme de mémoire collective se rattache au devoir de mémoire qui parcourt la trilogie de Nathan WACHTEL. Elle est « exemplaire » dans la mesure où elle se veut souvenir d'éléments, souvent traumatisants et donc indissociables du contexte inquisitorial. C'est ce devoir de mémoire qui est souvent privilégié par l'historiographie.

11 On sait que les souffrances vécues au nom du judaïsme ont justifié l'intégration des marranes à la diaspora et participent de cette culture du martyre qui est fondamentale dans la Nação. Les marranes posent en effet le sacrifice de soi comme un acte de foi ; la douleur devient un critère d'appartenance et de prestige au sein de la société judaïsante. Cela permet également de comprendre l'importance des jeûnes effectués avec grande rigueur durant tout le calendrier cultuel. Un certain nombre d'éléments stigmatisants sont renversés et deviennent des éléments de cet orgueil. Je pense en particulier au cas de Sambenitos qui peuvent se changer en objets de fierté. Par exemple, c'est le cas dans les années 1560 de Cristobal de BAENA, un petit-fils de condamné, dans la région de Jaén. Lorsqu'on lui parlait de la honte causée par la présence du Sambenito dans la cathédrale de la ville, il répondait: "c'est là mon drapeau » (esa es mi bandera). Signes de la macule judaïsante et judéoconverse, fardeaux 
pour les lignages, les sambenitos deviennent ainsi les marqueurs de la mémoire marrane et justifient leur existence en tant que groupe et peuple séparé.

De même que les marranes s'intègrent à la Nação, ils rétablissent une continuité historique primordiale pour effacer la rupture constituée par l'apostasie de leurs ancêtres à la fin du XV $\mathrm{XV}^{\mathrm{e}}$ siècle et la simulation du catholicisme ensuite. C'est pourquoi les marranes retissent les fils qui les relient à l'histoire juive, que ce soit par référence à l'âge d'or du judaïsme hispanique dans l'Al-Andalus dont ils seraient les héritiers directs ou par l'institution de généalogies fabuleuses qui les font descendre de figures qui ont marqué l'histoire d'Israël, David entre autres. D'autres se prétendent de filiation lévitique, d'autres encore issus des tribus de Juda et de Benjamin ou enfin des tribus perdues d'Israël que j'évoquerai plus tard. En effet, nous retrouvons le désir d'immortalité, d'antiquité mais aussi de noblesse ainsi que le destin messianique dans cette filiation.

13 La narration occupe donc chez les marranes une place centrale et détermine les moments qui marquent l'histoire du groupe. L'acte même de narrer est ritualisé et soude le collectif en instituant une expérience partagée. Le rappel des souffrances vécues par les ancêtres fait du souvenir un impératif moral. D'ailleurs, cela est assez présent dans la trilogie de Nathan WACHTEL. Les parallèles entre marranes et Hébreux, notamment, faisant revivre aux premiers les souffrances des seconds, sont omniprésents dans les témoignages. Par exemple, Maria LOPEZ vivant à Osuna au début du XVII e siècle explique les jeûnes des «trois semaines » (un moment du calendrier cultuel) en ces termes : Il ne fallait, disait-elle, « rien faire ni s'engager dans quelque tâche que ce soit, ni ouvrage parce cela se terminait mal [«no tenia buen fin»] du fait d'une guerre qui avait eu lieu à l'époque avec les Hébreux, durant laquelle ils [les Hébreux] avaient été très malheureux et beaucoup de sang était tombé ».

En inscrivant leurs récits dans le contexte biblique, les marranes s'efforcent, sinon de les rendre vraisemblables, du moins de leur conférer une aura sacrée et un sens d'ordinaire aux accents messianiques.

Ces récits constituent, pour l'historien, une aubaine. Ce sont en effet les lieux où, dans des textes extrêmement normés que sont les sources inquisitoriales, en particulier les procès de foi, il peut tenter d'approcher les pratiques sociales et cultuelles marranes.

II. Mon second point concerne la lecture des sources inquisitoriales. La foi du souvenir et La logique des bûchers (les deux premiers tomes de la trilogie), sont structurés de manière analogue : par portrait et par cas. Chaque chapitre est d'ordinaire consacré à un ou deux cas, des trajectoires. Carlo SEVERI parlait justement de ces biographies, de ces trajectoires et de ces « labyrinthes » du premier volume donnant à voir une part du collectif. Cela répond à la volonté de Nathan WACHTEL de déplacer le regard pour restituer "le point de vue des vaincus ", d'une histoire à la fois vue d'en bas et dans le monde souterrain révélant ainsi le quotidien du monde marrane.

Ces enquêtes "micro-historiques", comme Nathan WACHTEL les qualifie, correspondent avant tout à la structuration des archives et des procès inquisitoriaux. Je vous rappelle que l'une des singularités des inquisitions ibériques réside dans le fait que, face à la multiplicité des territoires et des unités juridictionnelles, elles apparaissent alors comme des modèles de centralisation et de discipline bureaucratique. Je rejoins sur ce point Nathan WACHTEL qui, dans l'introduction de $L a$ logique des bûchers,parlait justement d'une « modernité de l'Inquisition ». 
18 En effet, les inquisitions sont parmi les seuls organes ayant réussi à étendre leur mainmise sur l'ensemble des territoires ibériques et participent en ce sens à la structuration des empires. Ainsi, les inquisitions ont développé un ample réseau d'information trans-océanique indispensable pour suivre les accusés particulièrement mobiles dans le cas des judéoconvers, ce qui contribue à la grande richesse de la documentation. On sait en effet que l'inculpation d'un membre d'une complicité (complicidad), c'est-à-dire les personnes associées dans un même acte d'accusation permet d'ouvrir des dossiers et d'accumuler les preuves contre le reste du groupe.

Plus largement, l'ensemble des activités des tribunaux locaux était soumis au regard du pouvoir central et engendrait donc une correspondance intense avec les organes centraux à Madrid ou à Lisbonne dans le cas du Brésil. Mais si les archives inquisitoriales sont particulièrement riches, elles sont lacunaires. Nous n'avons généralement que des relations de cause et de procès, excepté pour certains tribunaux pour lesquels on dispose des procès complets, en particulier ceux de Mexico et de Lisbonne qu'utilise principalement Nathan WACHTEL dans sa trilogie.

La démarche qu'il adopte dans les deux premiers volumes est assez similaire : le résumé des procès, des trajectoires, introduit un large recours aux citations pour, dit Nathan WACHTEL, "donner à entendre [...] les voix de nos témoins». D'ailleurs, selon un procédé analogue, toute une partie du troisième volume est consacrée à ces « récits de vie » de juifs marranes du nord-est brésilien contemporain.

21 Ces longs procès sont à la fois une chance, mais également un risque du fait de la nature des sources inquisitoriales. Toute la question, en effet, qui a fait l'objet de nombreuses controverses parmi les spécialistes, est de savoir quel est le crédit que nous pouvons donner à ces documents pour connaître les pratiques des individus. C'est évidemment un problème que se pose tout historien et, en particulier, ceux qui travaillent sur des sources judiciaires.

Il y a toutefois une certitude: toutes les charges ne sont pas inventées, comme le postulaient certains historiens, en particulier Antonio SARAIVA au siècle dernier. Pour autant, on ne peut arguer que "l'inquisition ne se trompe jamais » comme l'avançait Israël REVAH, le grand historien des marranes. Dès lors, la question est de savoir à quel point, comment? Si la rigueur du Saint-Office est bien réelle comme sa sévérité à l'égard des faux témoignages, l'attente des inquisiteurs structure indubitablement les propos des témoins-accusés.

23 D'ailleurs, cela est manifeste dans les instructions données aux tribunaux. Je prendrai l'exemple de l'interrogatoire-type dont disposaient les inquisiteurs de Murcie au début du XVIII ${ }^{e}$ siècle, soit les dix-huit «questions qui sont posées aux accusés de ce délit après avoir confessé ». Parmi ces dix-huit questions, six portent sur les sacrements, quatre sur les autres cryptojudaïsants. Toutefois, aucune question ne s'intéresse directement aux rites suivis.

De même, les édits de foi (donc les textes lus aux fidèles ou affichés) recensant les rites judaïques prohibés n'ont pratiquement pas changé du $\mathrm{XVI}^{\mathrm{e}}$ au XVIII ${ }^{\mathrm{e}}$ siècle. Cela explique qu'ils aient pu servir de source d'information pour les marranes eux-mêmes. Par la même, on comprend que des pans entiers de la vie des accusés soient passés sous silence, en particulier les relations avec leurs vieux-chrétiens et que les aveux soient si répétitifs surtout au sein d'une même « complicité ». 
25 En réalité, on constate que tout ce qui sort du cadre, de l'horizon d'attente, pose problème. C'est le cas des unions entre les judéoconvers judaïsants et les vieuxchrétiens catholiques. Pour preuve la lettre du tribunal de Murcie en 1721 au Conseil de l'Inquisition à Madrid (l'organe central) à propos des allégations de Beatriz VAZQUÉZ contre différentes personnes dont un certain Sébastian GARCIA. Les inquisiteurs soulignent que : "Presque toutes les personnes que met en cause Béatriz sont de vieuxchrétiens et Sébastian qui est de caste de juifs [...] n'aurait aucune raison de s'opposer à ce témoignage [celui de Béatriz] s'il était vrai. À cela s'ajoute l'invraisemblance du contenu du témoignage de cette Beatriz, vu que ce ne sont pas des cérémonies que font habituellement les observants [judaïsants]».

Deux critères sont ici déterminants. Tout d'abord, que les témoignages à charge entrent ou non dans la grille inquisitoriale. Ensuite, la filiation d'un accusé, c'est-à-dire son ascendance, décide souvent de la nature du délit. Dans nombre de cas en effet, on constate que c'est le regard inquisitorial qui classe ces hérésies. Autrement dit, les mêmes éléments seront qualifiés soit de crypto-judaïsme soit de crypto-islamisme suivant qu'il s'agisse d'un judéoconvers ou d'un morisque (chrétien d'origine musulmane).

Par conséquent, les témoignages sont triplement biaisés. D’abord, par les conditions d'énonciation, puisque les accusés peuvent mettre en œuvre des stratégies de défense voire se concerter. Ensuite, par l'attente des inquisiteurs qui se reflète dans les interrogatoires. Enfin, par les catégories de pensées du scripteur (le secrétaire inquisitorial) qui influent sur la transcription des mots du témoin.

Toute la difficulté pour l'historien est de lire les écarts à la norme lorsque les témoignages sortent de la grille inquisitoriale et des stéréotypes des inquisiteurs, échos de leurs questions et des édits de foi. D'ailleurs, Nathan WACHTEL adopte notamment cette démarche dans La logique des bûchers en utilisant les descriptions effectuées dans lesdites " cárceles de vigia ", c'est-à-dire les cellules d'observation dans lesquelles les prisonniers sont observés à leur insu par un trou. Les agents se relaient et notent tout ce qu'ils voient. Toutefois, on constate que là encore l'interprétation est difficile, puisque les observateurs comprennent et interprètent ce qu'ils voient par rapport à leurs propres référents. Je pense par exemple aux jeûnes qu'aurait effectué Miguel de MENDONÇA dans les prisons de Lisbonne en 1729 et à l'importance accordée par les agents de savoir si " la première étoile était ou non sortie ", élément mis en avant dans les édits de foi pour déterminer s'il s'agissait bien d'un jeûne.

29 Certes, je l'ai dit, les édits de foi ont pu constituer une source d'information non seulement pour l'Inquisition et ses agents, mais également pour les marranes euxmêmes. Car, à mon sens, on ne saurait dissocier le phénomène marrane de son rapport à l'Inquisition.

III. Je souhaitais donc pour finir évoquer la question des résurgences marranes dans la longue durée et en particulier à l'époque contemporaine, aux $\mathrm{XIX}^{\mathrm{e}}$ et $\mathrm{XX}^{\mathrm{e}}$ siècles. L'un des objets de la trilogie, comme dans le reste de l'œuvre de Nathan WACHTEL, est d'établir « un lien entre le passé et le présent de l'extrême fin du XX ${ }^{\mathrm{e}}$ siècle pour rendre compte de phénomènes de résurgence [du marranisme] qui se produisent jusqu'à nos jours ». Ainsi, Nathan WACHTEL s'attache à détecter la persistance non tant de pratiques judaïques que "d'un sentiment intime », un « devoir de mémoire », comme il ressort de La foi du souvenir, dans la construction identitaire de ceux qui se revendiquent aujourd'hui « juifs marranes », une notion bien sûr problématique. 
31 L'analyse de ce marranisme contemporain, essentiellement dans le troisième volume, soulève de nombreuses questions.

Par exemple, on pourrait s'interroger sur les conditions d'énonciation des pratiques dont se souviennent les témoins. Je pense notamment au cas de Paulo VALADARES, dans l'épilogue de $L a$ foi du souvenir,rapportant avoir lu des ouvrages sur le marranisme en particulier ceux d'Anita NOVINSKY, dont on sent nettement la marque dans son récit. Cela est particulièrement notable lorsqu'il évoque les pratiques endogames et en particulier les mariages entre oncle et nièce et qu'il précise : "C'est une coutume qui date du temps de l'inquisition. La seule façon de garder le secret dans la famille ». Or il s'agit d'un topos de la première historiographie marrane qui a été depuis largement relativisé.

On s'interroge également sur la nature de ces "résurgences». Marquent-elles une forme de permanence, de continuité depuis le $\mathrm{XV}^{\mathrm{e}}$ siècle? Ou alors s'agit-il d'un réinvestissement de traces souvent inconscientes et d'une réinvention-création? On pourrait ainsi arguer que ces " résurgences », ces mouvements marranes dans le Brésil des années 1990 répondent à bien des égards à un besoin d'appartenance, une aspiration à la différence, qui semble d'ailleurs étroitement liée à la reconstruction généalogique et que l'on perçoit dans les motifs réitérés dans les témoignages. Un processus que Nathan WACHTEL présente comme «le sentiment originel d'une différence [...], la quête souvent douloureuse d'une explication, la découverte lumineuse de l'ascendance juive ». Ce besoin d'ailleurs, et cela me parait significatif, se traduit aussi chez les "juifs marranes" brésiliens par le recours aux tribus perdues d'Israël que nous trouvions déjà aux XVI ${ }^{\mathrm{e}}$ et XVIII ${ }^{\mathrm{e}}$ siècles.

Il faut bien voir dans quel contexte se situe cette redécouverte ou cette naissance du mouvement marrane. En effet la "découverte ", dans les années 1920, des «marranes de Belmonte ", dans le nord du Portugal, et la publication du livre de Samuel SCHWARZ, rappellent à bien des égards la recherche des tribus perdues qui avait déjà fait trembler la diaspora au XVII ${ }^{\mathrm{e}}$ siècle avec la soi-disant découverte d'Indiens judaïsants dans la région de Quito, censés être l'une des tribus perdues et que l'on retrouve notamment pour les Falashas d'Éthiopie ou les populations du nord de l'Inde, censées descendre de la tribu de Manassé.

En effet, c'est toute une aspiration que l'on voit clairement dans le troisième volume expliquant le fait que ces juifs marranes ne se contentent pas d'une conversion au judaïsme à laquelle étaient pourtant soumis les marranes des $\mathrm{XVI}^{\mathrm{e}}$ et $\mathrm{XVIII}{ }^{\mathrm{e}}$ siècles lorsqu'ils arrivaient dans une communauté juive et qu'ils insistaient pour parler d'un retour. La conversion leur apparait comme une forme de reniement du sacrifice de leurs ancêtres et un refus de légitimation de leur filiation. Je citerai à ce titre le témoignage d'Odmar PINHEIRO pour qui «Ceux qui se convertissent ne sont pas véritablement juifs; ils n'ont été que judaïsés, ils ne sont pas juifs en reconnaissance de leur origine, de leur tradition ".

La question est justement de savoir si ce soi-disant marranisme contemporain s'inscrit dans une continuité. En somme, peut-on dire que les traces de pratiques judaïques souvent sans réelle conscience d'après les témoignages et en l'absence de répression, peuvent être qualifiées de cryptojudaïsme? Et je renvoie ici au titre de mon intervention. 
37 En effet, à mon sens, le marranisme, va au-delà du maintien de formes de rituels, et d'une mémoire des origines mais constitue un « fait social total » dans lequel le rapport à l'Inquisition est, je l'ai dit, central. Il détermine aussi bien les pratiques sociales, les modes d'identification que l'intensité du secret. Les marranes forment plus qu'un ensemble d'individus partageant une croyance. Ils forment véritablement des unités sociales collectives, des communautés imaginées ayant un tissu social et une histoire propres. La conscience de soi et l'identification sont des aspects centraux, puisque le marrane est avant tout celui qui se perçoit et se présente comme tel mais surtout qui est réputé comme tel. Ainsi, les marranes sont rarement seuls, car ils s'insèrent dans des réseaux clientélaires et dans des solidarités familiales. L'individu prend sens au sein du collectif soudé par le secret et qui a multiplié les signes d'appartenance et les codes de langage. La clandestinité y est véritablement "performative ", elle garantit la sécurité des individus face au Saint-Office et légitime l'existence du groupe, fondant son identité collective. En ce sens, les communautés marranes correspondent aux sociétés secrètes analysées par SIMMEL.

En définitive, la solution pour interpréter ces résurgences, et le «marranisme contemporain ", serait de différencier deux types d'utilisation du terme " marranisme », deux formes distinctes : une utilisation située et une utilisation que je qualifierais de métaphorique.

Pour ce qui est de l'utilisation située tout d'abord. Le marranisme ibérique est un phénomène à mon sens temporellement et spatialement situé. En ce sens, je fais par exemple une distinction entre le marranisme ibérique soumis à l'Inquisition à d'autres cas de crypto-judaïsme que l'on trouve à la même époque en Angleterre, en France ou dans les Pays-Bas espagnols où la répression est effacée voire inexistante. Ces groupements, généralement en contact avec les congrégations juives officielles, sont d'ordinaire qualifiés de marranes par l'historiographie, souvent dans une perspective théologique renvoyant à leur institutionnalisation postérieure. Il convient donc de dégager des degrés ou des types de crypto-judaïsme dont le marranisme, dans son modèle ibérique, serait le dernier échelon. La distinction résiderait notamment dans une forme de reconnaissance tacite de ces communautés, dans laquelle la clandestinité n'est pas tant une protection face au danger, qu'une invisibilité pour se conformer aux normes socio-politiques qui régissent nombre de sociétés modernes à l'époque, une orthodoxie de façade en somme. Il s'agit d'une autre utilisation de la clandestinité dans laquelle le secret perd sa fonction structurante au profit d'un usage plus ponctuel.

Ensuite, une seconde utilisation, que j'ai qualifiée de métaphorique, renverrait en premier lieu à la généralisation du concept de "marranisme " comme un outil heuristique dans les sciences sociales où la «condition marrane » est sortie de son contexte historique comme métaphore d'une identité ambivalente ou annonçant, par la dissociation entre espaces public et privé, l'appartenance non cultuelle à une communauté ou une forme de modernité politique.

41 Par ailleurs, je l'utiliserai également pour analyser les traces du crypto-judaïsme aux $\mathrm{XIX}^{\mathrm{e}}$ et $\mathrm{XX}^{\mathrm{e}}$ siècles. Ce sont ces traces que Nathan WACHTEL qualifie de " réminiscence semi-consciente, savoir longtemps ignoré qui, lorsqu'il affleure à la conscience, revêt aussitôt l'évidence du toujours su ». Je pense ici au témoignage d'Isabel A. de Recife qui, à propos des pratiques «judaïsantes » de ses parents, déclare : « Mes parents vivaient tout cela sans savoir »; « on fait comme ça parce qu'on doit faire comme ça ». 
conclure, je souhaiterais souligner l'une des vertus de cette trilogie marrane, en l'occurrence la place laissée à l'émotion et aux sentiments qui sont d'ordinaire oblitérés par les historiens de l'Inquisition dont je fais partie. Celle, en somme, d'être une histoire vivante. Comme Carlo le précisait, c'est un regard qui note au détour d'un document la signature d'un accusé qui "paraît tremblante et toute rabougrie " ( $L a$ logique des bûchers). Les trois volumes se lisent comme des romans suivant le fil des figures qui ont marqué leur auteur à l'image d'Odmar PINHEIRO et BRAGA, le guide de Nathan WACHTEL au Brésil. C'est également une histoire militante, celle d'une forme de résistance de ceux qui ne s'avouent pas vaincus face à une machine inquisitoriale analysée en analogie avec les systèmes totalitaires contemporains. Cette histoire militante et vivante qu'illustrent avec force les photographies de l'exposition sur « L'art marrane dans le Sertão " achevant le troisième volume. Je vous remercie de votre attention. (Applaudissements).

\section{RÉSUMÉS}

Le phénomène marrane, la perpétuation de pratiques judaïsantes parmi les descendants des juifs convertis au christianisme dans les empires ibériques, et son pendant antagoniste, l'Inquisition, sont au cœur de l'œuvre de Nathan Wachtel depuis 2001. Dans les trois ouvrages et les articles qui ont marqué ce nouveau volet d'analyse, il s'essaie, à travers les archives abondantes mais équivoques du Saint-Office, où se lisent les méandres des trajectoires individuelles, à relier passé et présent, mémoire d'une identité originelle et effacement face à la stigmatisation et à l'oubli. Le marranisme est ainsi devenu, depuis une trentaine d'années, et en partie grâce à lui, un concept analytique récurrent dans les sciences sociales pour penser celui qui se déconstruit toujours luimême ou bien la condition de "l'homme moderne", divisé entre le citoyen et l'homme privé. Pourtant, ce "fait social total», tel que le désigne N. Wachtel, est doublement situé, dans le temps et dans l'espace, indissociable de l'action répressive de l'Inquisition et des clivages qui traversent les sociétés ibériques des $16^{\mathrm{e}}-18^{\mathrm{e}}$ siècles. Il s'agit donc, en somme, d'interroger la pertinence de la généralisation de la « condition marrane » dans la longue durée comme un outil heuristique des sciences sociales.

\section{AUTEUR}

\section{NATALIA MUCHNIK}

CRH, EHESS 